\title{
Editorial
}

\section{Medical ethics and medical education}

Should we be teaching medical ethics in British medical schools or not? If not, why not? If so, how? These questions are among the many important ones raised by Ian Kennedy in his Reith Lectures and discussed by various commentators in this issue of the fournal, an issue devoted entirely to analysis and criticism of those lectures, including Kennedy's detailed replies to each critic.

A common response from medical teachers when asked these questions is that of course medical ethics should be taught and that it is being taught, both within the curriculum and without. Thus in 1967 the General Medical Council recommended that medical ethics should be taught as part of the basic medical education ( $\mathrm{I}$ ) and in 1974 a GMC survey revealed that 25 out of 34 medical schools had formal arrangements for teaching medical ethics and II of the 25 had a staff member specifically charged with the responsibility for teaching the subject (2). Furthermore all or almost all medical schools in the United Kingdom have medical groups affiliated to the Society for the Study of Medical Ethics (which also founded and publishes this journal). These groups are run by medical and some other health students with administrative and additional help from the Society and interested professionals. They provide a forum for discussion of medico-moral matters by means of lectures, symposia, small seminars and full scale conferences. The groups are totally independent of, but welcomed by, the medical school authorities who to a small but increasing extent offer financial support. Finally, it is asserted, throughout their medical training medical students are exposed to medical ethics in action - to the moral decisions and actions of their teachers made within a professional ethos which embraces and transmits high standards of medical ethics.

Are then the current provisions comprised in these three different strands of British medical ethics teaching sufficient?

So far as the third strand is concerned - the exposure of students to medical ethics in action - it is important to differentiate between various uses of the term 'ethics' (3). Thus inculcating norms of behaviour and attitude which conform to the dominant ethos of medical practice is teaching medical ethics in one sense of the term - but it is not teaching medical ethics in the primary sense of the term, which embraces the critical study of moral decisions, attitudes, actions and norms. Such analytic medical ethics includes the distinguishing of moral from technical issues, the recognition and evaluation of different strategies for responding to moral issues, and learning to deal with moral conflict, whether it arises within an ethical theory or results from a clash of theories. Such medical ethics encourages not the inculcation of uncriticised moral norms but the development of a personal moral stance established as a result of critical analysis of alternatives.

Is such analytic medical ethics an appropriate part of medical education? Two separate lines of objection argue that it is not. On the one hand doctors may claim that in general doctors should simply be taught to behave according to the norms of the profession. The norms themselves have 'stood the test of time' (the Hippocratic Oath still affords their basis) and as new problems arise to cast doubt on these norms (for example over abortion) or to indicate the need for additional norms (for example concerning experimentation on human subjects) members of the profession who are interested, experienced and senior should ponder together, taking advice as they deem appropriate, and provide amplification or revision of the existing norms. In such a way evolved the various World Medical Association Declarations of Geneva, Helsinki, Sydney, Oslo, Tokyo and Hawaii.

The second line of objection is represented in the paper in this issue from the Chairman of the British Medical Association's Ethics Committee. He suggests that more teaching in medical ethics would be unnecessary if the public did as they should and laid down the broad moral guidelines within which they wished the medical profession to practise. Both objections carry the implication that for most doctors, "theirs not to make reply, theirs not to reason why'.

There are of course important counterarguments to these objections. Perhaps one of the strongest is that doctors should be mature moral agents and that all mature moral agents should make their moral decisions on the basis of principles which they have critically espoused rather than relying on principles established on their behalf by others. A second, and 
perhaps to some doctors more appealing, counterargument is that uncritical acceptance of others' principles, attitudes and behaviour no longer suffices in clinical practice. Patients in our plural society themselves have various norms and expectations and are often unwilling to accept the moral standards and decisions of their doctors, at least without adequate discussion. Even when they are willing, the nature of medical ethical problems is sometimes just too complex for their proper resolution to be even attempted let alone achieved on the basis of Oaths, Declarations, and inculcated professional norms (take for example the problems posed by severely handicapped newborn infants). Certainly where doctors believe it proper to make such complex moral decisions on behalf of their patients, either because the patients have asked them to do so or because they believe it their duty to do so unasked, the argument that they should be educated to understand and analyse the issues involved becomes compelling.

Given that the teaching of analytic medical ethics is desirable in British medical schools, is it best provided by voluntary largely student-run groups such as the London and other medical groups, or by formal instruction within the medical curriculum or by both? Kennedy, in his reply in this issue to the student president of the London Medical Group (who himself calls for formal curricular education in medical ethics) suggests that the medical groups may be a Trojan Horse. His reasoning is that they allow medical school authorities to wash their hands of the responsibility of providing adequate medical ethics teaching on the grounds that the medical groups are doing it already. Kennedy points out that the medical groups, admirable as they are, only reach those students who voluntarily attend their meetings in their spare time. Trojan Horse is hardly the appropriate term in this context for organisations which have pioneered the analytic and interdisciplinary discussion of medical ethics in British medical schools. It cannot be denied, however, that members of an important segment of the British medical student population only rarely or never give up their free time to attend such groups - a segment which arguably contains students who are the most in need of education in the principles of analytic medical ethics.

Finally, while it is difficult to be sure what the situation is today in regard to formal medical ethics teaching in Britain as compared with that in 1974 reported by the GMC, there is no doubt that there are still medical schools which provite none at all - and the extent of that which is provide $\mathrm{d}$, varies considerably.

There seems to be at least a prima facie case $\overrightarrow{\text { 趈or }}$ the claim that some sort of formal and probably compulsory teaching of analytic medical ettücs should be provided within the medical curriculsim in addition to exposure of medical students to ghe voluntary work of the medical groups and to the medical-ethics-in-practice manifested by their teachers. Whether or not thorough examination would sustain this prima facie case should be put to the test-by examining it thoroughly. The General Medical Council has already stated t角at medical ethics should be taught. Would it not? sensible for representatives of all the British medical schools to discuss, perhaps initially in the context of a conference, whether or not matters are satisfactory as they are? If not they might perhaps establish a working party to consider in some depth how best to improve them. As well ${ }_{\text {das }}$ pooling experience of medical ethics teaching@ึin Britain they might seek advice from medieal schools in other countries, especially in the U\$A, where analytic medical ethics has become established within medical curricula: their varied experieace may provide important warnings as well as more positive assistance.

Two responses should not be allowed to fois a proper investigation. The first is the stock co 1 plaint about current economic circumstangs. The second is that there is no room in the medieal curriculum for additional subjects. First, analyic medical ethics is a low technology, low cost subje ct and it may well be possible to accommodate it wh relatively little disturbance to existing curricala and budgets. Second, and more important, one the fundamental questions to be decided is whether or not instruction in analytic medical ethics is a necessary part of medical education. To state that while it may be desirable it cannot be afforked or cannot be fitted in is to beg this fundamental question, not to answer it.

\section{References}

(I) General Medical Council. Recommendation as?to basic medical education. London: GMC, 1967: 8.

(2) General Medical Council. Survey of basic medreal education in the British Isles. London: Nuffiegld Provincial Hospitals Trust, 1977.

(3) $C f$. Downie $\mathrm{R}$ S. Ethics, morals, and mefal philosophy. Fournal of medical ethics $1980 ; 6: 33-\Phi 4$. 